\title{
Carcinoma Lung Presenting as Sister Mary Joseph's Nodule
}

\author{
Sant Prakash Kataria', Sucheta ${ }^{2 *}$, Renuka Verma ${ }^{3}$, Bharti Saklani ${ }^{4}$, Pooja Pawaria ${ }^{4}$, Sangeeta Bamel ${ }^{4}$ and \\ Rajeev Sen $^{5}$
}

${ }^{1}$ Professor, Department of Pathology, Pandit Bhagwat Dayal Sharma Post Graduate Institute of Medical Sciences, India

${ }^{2}$ Senior Resident, Department of Pathology, Pandit Bhagwat Dayal Sharma Post Graduate Institute of Medical Sciences, India

${ }^{3}$ Assistant Professor, Department of Pathology, Pandit Bhagwat Dayal Sharma Post Graduate Institute of Medical Sciences, India

${ }_{4}^{4}$ Junior Resident, Department of Pathology, Pandit Bhagwat Dayal Sharma Post Graduate Institute of Medical Sciences, India

${ }^{5}$ Senior Professor \& Head of Department, Department of Pathology, Pandit Bhagwat Dayal Sharma Post Graduate Institute of Medical Sciences, India

*Corresponding author: Sucheta, Department of Pathology, Pandit Bhagwat Dayal Sharma Post Graduate Institute of Medical Sciences, India.
Received Date: September 14, 2020

Published Date: October 01, 2020

\begin{abstract}
Metastatic nodules of the umbilicus from malignant neoplasms are known as Sister Mary Joseph's nodule and they represent an advanced malignancy. These nodules are mostly adenocarcinomas with the commonest primary sites in intraabdominal organs. Here, we describe case of a patient with squamous cell carcinoma of lung who presented with metastatic umbilical nodule as the initial presentation of the tumor. Metastatic umbilical nodules from lung carcinoma and that too from squamous cell carcinoma are very rare. Patients presenting with umbilical nodules should be investigated without any delay and metastatic nodule should be included in the differential diagnosis especially in elderly patients.
\end{abstract}

Keywords: Sister Mary Joseph's Nodule; Squamous cell carcinoma; Umbilical nodule

\section{Introduction}

Sister Mary Joseph's Nodule is the name given to the malignant metastatic deposits of the umbilicus. The term "Sister Mary Joseph's Nodule was coined by Sir Hamilton Bailey in his book, Physical Signs in Clinical Surgery, in the honor of Sister Mary Joseph who was a surgical assistant of Dr. William Mayo in the early days of the Mayo Clinic. Sister Mary Joseph was the one who identified the relationship between umbilical nodules and advanced intraabdominal malignancy. Metastases to the umbilicus is mostly from gastrointestinal tract or genitourinary tract tumors and they are usually adenocarcinomas. However, a squamous cell carcinoma of the lung metastasizing to the umbilicus is uncommon and has rarely been reported.

\section{Case Report}

We present a case of 56 years old male patient who came to the surgery clinic of our hospital with complains of a recently noticed nodule in the umbilical region. Patient was a thinly built male with blood pressure of $124 / 82 \mathrm{~mm} \mathrm{Hg}$, pulse rate of $78 / \mathrm{min}$ and temperature 37.2 degree Celsius. He was a chronic smoker with no other significant history and findings other than history of recent weight loss and increasing dyspnea. Physical examination revealed a firm and partially fixed umbilical nodule measuring $2 \times 1.5 \mathrm{cms}$. Overlying skin was erythematous and adherent to the nodule. Ultrasonography was advised to the patient which revealed a heterochronic lesion in the umbilical region. Fine needle aspiration 
of the nodule was performed for cytopathological examination. Smears prepared from the aspirate were stained and examined. Examination of smears revealed presence of atypical cells that were mostly singly scattered and few of them were forming small groups and clusters. Cells showed keratinization with the presence of few inflammatory cells in the background. It was reported as metastatic deposits from squamous cell carcinoma. The site of primary tumor was not known at the time of FNAC. After cytological reporting of the umbilical nodule the patient was further investigated. CT scan of the chest showed presence of a mass lesion in the right lung and CT guided biopsy was taken from the lesion. Histopathological examination of the biopsy revealed squamous cell carcinoma that was positive for p63 on immunohistochemistry (Figure 1,2\&3).

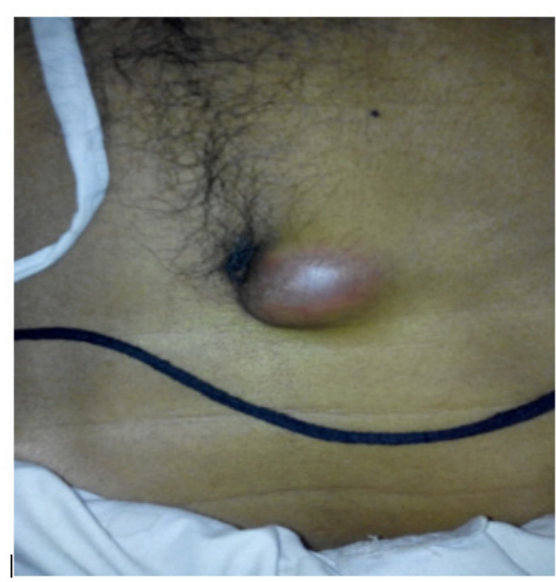

Figure 1: Umbilical nodule with overlying erythematous skin.

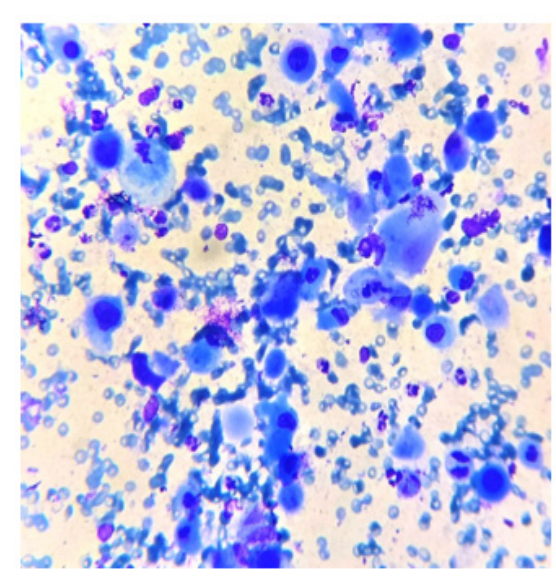

Figure 2: Photomicrograph showing atypical squamous cells on cytology (Leishman;400X).

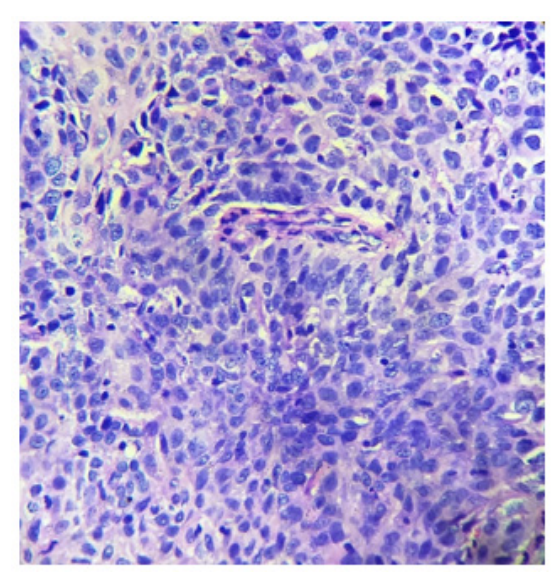

Figure 3: Photomicrograph showing invasive squamous cell carcinoma on histopathology (HE;400x). 


\section{Discussion}

Whenever a patient presents to the clinician with umbilical nodule the differential diagnosis inclu des both benign and malignant conditions. Benign conditions include endometriosis, melanocytic nevi, fibroepithelial polyps, papilloma's, dermatofibroma, fibroma, myoma, epithelial inclusion cyst, hemangioma, teratoma, pyogenic granuloma, urachal duct cyst, seborrheic keratosis, keloid, foreign body granuloma, myxoma, omphalitis, abscess, and umbilical hernia. Benign umbilical nodules are known as pseudo Sister Mary Joseph's Nodules. Malignant differential diagnosis includes both primary and secondary malignancy.

The occurrence of SMJN is uncommon and as a first sign of malignancy it is rare [1]. Gastrointestinal tract is the most common site of primary malignancy in patients with SMJN including stomach, colorectal and pancreas in decreasing frequency whereas in females epithelial tumors of ovary are the most common primary site followed by stomach, colorectal and pancreas and all of these include adenocarcinomas. Squamous cell carcinomas rarely metastasize to the umbilicus. Rare primary sites for metastasis to the umbilicus include gallbladder, liver, breast, lung, prostate, penis, peritoneum, lymphoma, bladder, and kidney. In some cases, the origin of the metastasis is unknown.

Diagnostic work-up of the patient should be done soon. Various imaging modalities can aid in the diagnosis like ultrasonography, computed tomography (CT), magnetic resonance imaging (MRI) and positron emission tomography (PET). Once SMJN are seen in a patient, FNAC or biopsy should be carried out immediately to reach the diagnosis. The architectural pattern and cellular morphology can aid in diagnosis of the underlying tumor. Immunohistochemistry can be used on histopathological sections to reach the type and origin of malignancy. Also, PET scan can aid in reaching the primary site of malignancy.

In our case the primary site came out to be lung and it was diagnosed as squamous cell carcinoma on biopsy. Lung carcinomas metastasize commonly to liver, adrenal glands, bone, kidney, and the central nervous system. The common cutaneous sites of metastasis from lung carcinoma are the chest wall and posterior abdomen but neck region can also be involved. However, the umbilicus is a rare site for metastasis from lung carcinoma and has rarely been reported [2-4].

The spread of malignancy to the umbilicus can occur in several ways. These include direct extension from a contiguous tumor, hematogenous, lymphatics and direct extension along the vestigial remnants of embryonal ligaments including the round ligament, the urachus, the Vitello intestinal duct remnant and the obliterated vitelline artery. Also, implantation after laparoscopy is another mode of spread of malignancy to umbilicus. Lymphatic drainage system of the umbilicus represents possible routes by which metastatic tumor cells can implant into the umbilical region.

In our case the patient first presented with an umbilical nodule and on receiving the cytopathological report of squamous cell carcinoma, further investigations were done to look for a primary site, which revealed squamous cell carcinoma of the lung. This is a rare and interesting presentation.

Sister Mary Joseph's nodule usually represents advanced malignancy and hence treatment is mainly palliative. Some authors have advocated wide excision with extensive search for the primary lesion. Some authors suggest radiotherapy and surgery with adjuvant therapy. As most of the patients with SMJN are inoperable, palliative therapy can improve the survival. Rest depends on the type of primary tumor and general health of the patient $[5,6]$.

\section{Conclusion}

Patients presenting with umbilical nodules should be examined carefully. Metastasis should be included as one of the differential diagnosis specially in elderly patients and diagnostic work up must be done as soon as possible so that appropriate line of treatment can be provided to the patient soon [7].

\section{Acknowledgement}

None.

\section{Conflict of Interest}

No conflict of Interest.

\section{References}

1. Gabriele R, Conte M, Egidi F, Borghese M (2005) Umbilical metastases: Current viewpoint. World J Surg Oncol 3(1): 13.

2. Panaro F, Andorno E, Di Domenico S, Morelli N, Bottino G, et al. (2005) Sister Joseph's nodule in a liver transplant recipient: Case report and mini review of literature. World J Surg Oncol 3(1):4.

3. Inamdar AC, Palit A, Athanikar SB, Sampagavi VV, Deshmukh NS (2003) Inflammatory cutaneous metastasis as a presenting feature of bronchogenic carcinoma. Indian J Dermatol Venereol Leprol 69(5): 347349 .

4. Idelvich E, Husar M, Fenig E, Brenner B, Ben-Baruch N, et al. (2000) Diagnostic Dilemmas in Oncology. J Clin Oncol 18: 3188-3189.

5. Saito H, Shimokata K, Yamada Y, Nomura F, Yamori S (1992) Umbilical metastasis from small cell carcinoma of the lung. Chest 101(1): 288-289.

6. Dutta U, Kumar M, Sharma SC, Nagi B (2004) Umbilical metastasis with squamous cell carcinoma of esophagus. Indian J Gastroenterol 23(4): 156-157.

7. Hamakawa H, Bao Y, Takarada M, Fukuzumi M, Tanioka H (1998) Cytokeratin expression in squamouscell carcinoma of the lung and oral cavity: An immunohistochemical study with possible clinical relevance. Oral Surg Oral Med Oral Pathol Oral Radiol Endod 85(4): 438-443. 